\title{
Serum potassium levels as an outcome determinant in acute medical admissions
}

\author{
Authors: Richard Conway, ${ }^{\mathrm{A}}$ Donnacha Creagh, ${ }^{\mathrm{B}}$ Declan G Byrne, ${ }^{\mathrm{C}}$ Deirdre O'Riordan ${ }^{\mathrm{D}}$ and Bernard Silke ${ }^{\mathrm{E}}$
}

\begin{abstract}
The relationship between serum potassium levels and mortality in acute medical admissions is uncertain. In particular, the relevance of minor abnormalities in potassium level or variations within the normal range remains to be determined. We performed a retrospective cohort study of all emergency medical admissions to St James's Hospital (Dublin, Ireland) between 2002 and 2012. We used a stepwise logistic regression model to predict in-hospital mortality, adjusting risk estimates for major predictor variables. There were 67,585 admissions in 37,828 patients over 11 years. After removing long-stay patients, 60,864 admissions in 35,168 patients were included in the study. Hypokalaemia was present in $14.5 \%$ and hyperkalaemia in 4.9\%. In-hospital mortality was 3.9, 5.0, and $18.1 \%$ in the normokalaemic, hypokalaemic and hyperkalaemic groups respectively. Hypokalaemic patients had a univariate odds ratio (OR) of 1.29 for in-hospital mortality ( $95 \%$ confidence interval (CI) 1.16-1.43; $\mathrm{p}<0.001$ ). Hyperkalaemic patients had a univariate OR for in-hospital mortality of 5.2 (95\% CI 4.7-5.7; $\mathrm{p}$ <0.001). The ORs for an in-hospital death for potassium between 4.3 and $4.7 \mathrm{mmol} / \mathrm{l}$, and 4.7 and $5.2 \mathrm{mmol} / \mathrm{l}$, were 1.73 (95\% CI 1.51-1.99) and 2.97 (95\% CI 2.53-3.50) respectively. Hyperkalaemia and hypokalaemia are associated with increased mortality.
\end{abstract}

KEYWORDS: Mortality, outcomes, potassium, emergency admissions

\section{Introduction}

Potassium homeostasis is critical to the maintenance of health and life. Significantly elevated $(>6.5 \mathrm{mmol} / \mathrm{l})$ or decreased $(<3 \mathrm{mmol} / \mathrm{l})$ potassium levels can result in adverse events ranging from muscle cramping to cardiac arrythmias and death. ${ }^{1,2}$ The correction of such life-threatening hypo- and hyperkalaemic states is basic medical knowledge taught to all

Authors: Aspecialist registrar, Department of Internal Medicine, St James's Hospital, Dublin, Ireland; Binformation analyst, Information Management Systems, St James's Hospital, Dublin, Ireland; C consultant physician, Department of Internal Medicine, St James's Hospital, Dublin, Ireland; ${ }^{D}$ consultant physician, Department of Internal Medicine, St James's Hospital, Dublin, Ireland; ${ }^{E}$ consultant physician, Department of Internal Medicine, St James's Hospital, Dublin, Ireland medical students and trainees. The optimum management of lesser degrees of potassium abnormalities or the use of supplementation in those with low-normal potassium values is less certain.

The artificial adjustment of potassium levels within the normal range, most typically potassium supplementation, to meet a pre-specified target potassium is common in clinical practice. Clinical practice guidelines following acute myocardial infarction recommend maintaining serum potassium between 4.0 and $5.0 \mathrm{mmol} / \mathrm{l}$ or even higher. ${ }^{3-5}$ While its origins lie in the management of acute cardiac cases, this phenomenon has spread, initially to other critically ill patients in the intensive care unit (ICU) and coronary care unit (CCU), and subsequently to general medical patients with non-cardiac conditions. ${ }^{6}$ This may be due to a diffusion of knowledge of this practice from the cardiac and intensive care wards. Two issues arise with this practice. The first, is there any evidence for the practice of routinely supplementing potassium to attain high-normal levels? The second, what is the effect of this practice when performed on general medical wards outside the close clinical and biochemical scrutiny of an ICU or CCU? Supplemental potassium administration is the most common cause of severe hyperkalaemia in hospitalised patients. ${ }^{2}$

The recommendations for maintaining a high-normal serum potassium arose from a series of trials showing evidence for an increased risk of ventricular arrhythmia in hypokalaemic patients post myocardial infarction. ${ }^{7-9}$ These trials were conducted prior to the advent of many modern therapies and their applicability to modern medical care is uncertain. ${ }^{6}$ Recent evidence in patients with acute myocardial infarction shows that the lowest mortality is found in those patients with a serum potassium between 3.5 and $<4.5 \mathrm{mmol} / \mathrm{l}^{6}$

The aim of this study was to assess the association between serum potassium levels and in-hospital mortality in the acute medical assessment unit (AMAU) setting.

\section{Material and methods}

\section{Background}

St James's Hospital serves as a secondary care centre for emergency admissions for its local Dublin catchment area of 270,000 adults. Emergency medical patients are admitted from the emergency department to an AMAU opened in 2003, the operation and outcome of which have been described elsewhere. ${ }^{10}$ 
This retrospective cohort study enrolled all patients admitted medically through the emergency department between January 2002 and December 2012, including patients admitted to the ICU or high dependency unit. A shortage of nursing home beds and resources means that $50 \%$ of all acute bed utilisation in our institution is attributable to just $11 \%$ of episodes. In order to avoid a disproportionate representation of these 'non-medical' admissions in our results we limited our dataset to those patients who were discharged or suffered an in-hospital death within 30 days. Patients admitted under services other than general medicine were excluded from the analysis.

\section{Data collection}

For audit purposes we employed an anoymous patient database, assembling core information about each clinical episode from elements contained on the patient administration system, the national hospital in-patient enquiry scheme, the patient electronic record, the emergency room and laboratory computers. ${ }^{11}$

Data held on the database includes the unique patient number, admitting consultant, date of birth, gender, area of residence, principal and up to nine additional secondary diagnoses, principal and up to nine additional secondary procedures, and admission and discharge dates. Additional information crosslinked and automatically uploaded to the database includes admission physiological, haematological and biochemical parameters.

We used discharge codes to calculate the Charlson comorbidity index to evaluate comorbidity. ${ }^{12}$ Deprivation status was calculated based on the SAHRU national deprivation index..$^{13}$ The urgency of each case presenting to the emergency department was assessed using the Manchester triage category. ${ }^{14}$ Disabling disease score has been demonstrated to be a predictor of mortality and therefore has been used as a risk adjustor in our multivariate model. ${ }^{15,16}$ We have developed an acute illness severity score based on serum sodium, potassium, urea, albumin, red cell distribution width and white blood cell count, that predicts clinical outcomes. This has been used as a risk adjustor in our multivariable model. ${ }^{17,18}$

The normal range for potassium was considered to lie between 3.5 and $5.0 \mathrm{mmol} / \mathrm{l}$. Based on the frequency distribution, potassium was considered as a continuous and categorical (7 group) variable $(<2.5, \geq 2.5<3.0, \geq 3.0<3.5, \geq 3.5<4.3$, $\geq 4.3<4.7, \geq 4.7<5.2$, and $\geq 5.2 \mathrm{mmol} / \mathrm{l})$. We further analysed potassium as hypokalaemia $(<3.5 \mathrm{mmol} / \mathrm{l})$, normokalaemia and hyperkalaemia ( $>5 \mathrm{mmol} / \mathrm{l})$. Potassium was unknown in $5.3 \%$ of those presenting. In this case it was assumed normal and a random value in the normal range was allocated. Missing data for other parameters were not imputed and data were analysed on the available full dataset, which was present in $74 \%$ of admissions.

\section{Statistical methods}

Descriptive statistics were calculated for background demographic data. Comparisons between categorical variables and mortality were made using chi-square tests. The association between in-hospital mortality and serum potassium level, adjusted for age-adjusted acute illness severity score, ${ }^{17,18}$ the Charlson comorbidity score ${ }^{12}$ disabling disease score,${ }^{15} \mathrm{O}_{2}$ saturation and Manchester triage status, ${ }^{14}$ was examined. Logistic analysis of the binomial outcome variable was undertaken by fitting a generalised estimating equation (GEE) regression model that allowed for clustered data; this is necessary to account for correlations between observations for a given subject (readmissions). ${ }^{19,20}$ We calculated the predicted probabilities after fitting the GEE model and used these values to derive a quadratic plot with its confidence intervals from the model. For length of stay (LOS), we employed a zero-truncated Poisson regression model..$^{21}$ We used robust standard errors for the parameter estimates. ${ }^{22}$

Adjusted ORs and 95\% CIs were calculated for those predictors that significantly entered the model $(\mathrm{p}<0.10)$. $\mathrm{p}<0.05$ was considered to indicate statistical significance. Stata v.12.1 (Stata Corporation, College Station, TX, USA) statistical software was used for analysis. This study was approved and conducted in accordance with local institutional review board guidelines.

\section{Results}

\section{Patients}

A total of 67,585 admission episodes were recorded in 37,828 unique patients admitted acutely between January 2002 and December 2012. After removing long-stay patients, a total of 60,864 episodes in 35,168 unique patients remained. Baseline characteristics of the population were calculated per admission. The median (interquartile range (IQR)) LOS was 5.0 (2.0-9.8) days. The median (IQR) age was $62.0(41.8-76.9)$ years. The proportion of males was $49.1 \%$. A Charlson comorbidity score of 0,1 or $\geq 2$ was present in $45.5,27.1$ and $27.4 \%$ respectively. Hypokalaemia was present in $14.5 \%$ and hyperkalaemia in $4.9 \%$ of patients. Hypokalaemic patients were significantly younger than normokalaemic patients at admission - median (IQR) 58.1 (40.8-74.7) years versus 61.9 (41.3-76.8) years $(\mathrm{p}<0.001)$. Their Charlson index (1.49 vs 1.47$)$ and disability scores (3.4 vs 3.36) were similar (Table 1). Hyperkalaemic patients were significantly older, median (IQR) 73.8 (57.3-82.2) years compared with normokalaemic patients, median (IQR) $61.9(41.3-76.8)$ years $(\mathrm{p}<0.001)$. There was a higher proportion of patients with Charlson index grade $\geq 2$, disability scores 3 and 4 , and elderly (75+) and very elderly (85+) in the hyperkalaemia cohort (Table 1). Hospital mortality by admission was $4.8 \%$ (95\% CI 4.6-5.0). The median (IQR) time to death was 7.0 (2.5-15.0) days.

Overall there was a curvilinear relationship between admission potassium and in-hospital mortality; these data suggested that hyperkalaemia was more likely to lead to death (Fig 1).

\section{Association of hypokalaemia with mortality and hospital length of stay}

Patients with hypokalaemia at admission had a higher inhospital mortality (5.0\%) compared with those normokalaemic at admission $(3.9 \% ; \mathrm{p}<0.001)$ (Table 1$)$. The univariate OR for in-hospital mortality for hypokalaemia versus normokalaemia was 1.29 (95\% CI 1.16-1.43; p<0.001). When adjusted for other predictive risk factors, including illness severity score, Charlson comorbidity index, Manchester triage admission category, 
Table 1. Emergency admissions (2002-2012) by admission potassium.

\begin{tabular}{|c|c|c|c|c|c|}
\hline Factor & Normokalaemia & Hypokalaemia & $p$ value $^{a}$ & Hyperkalaemia & p value ${ }^{b}$ \\
\hline Patients, $\mathrm{n}$ & 49,087 & 8,811 & & 2,966 & \\
\hline \multicolumn{6}{|l|}{ Gender } \\
\hline Male & $24,601(50.1 \%)$ & $3,705(42.0 \%)$ & \multirow[t]{2}{*}{$<0.001$} & $1,548(52.2 \%)$ & \multirow[t]{2}{*}{0.028} \\
\hline Female & $24,486(49.9 \%)$ & $5,106(58.0 \%)$ & & $1,418(47.8 \%)$ & \\
\hline \multicolumn{6}{|l|}{ Outcome } \\
\hline Alive & $47,152(96.1 \%)$ & $8369(95.0 \%)$ & \multirow[t]{2}{*}{$<0.001$} & $2430(81.9 \%)$ & \multirow[t]{2}{*}{$<0.001$} \\
\hline Died & $1,935(3.9 \%)$ & $442(5.0 \%)$ & & $536(18.1 \%)$ & \\
\hline Mean LOS, days (SD) & $6.8(6.5)$ & $7.6(6.6)$ & $<0.001$ & $8.4(7.0)$ & $<0.001$ \\
\hline \multicolumn{6}{|l|}{ Age group, years } \\
\hline $10-39$ & $11,549(23.5 \%)$ & $2,115(24.0 \%)$ & \multirow[t]{5}{*}{$<0.001$} & $335(11.3 \%)$ & \multirow[t]{5}{*}{$<0.001$} \\
\hline $40-59$ & $11,747(23.9 \%)$ & $2,540(28.8 \%)$ & & $499(16.8 \%)$ & \\
\hline $60-74$ & $11,734(23.9 \%)$ & $1,998(22.8 \%)$ & & $749(25.3 \%)$ & \\
\hline $75-84$ & $9,748(19.9 \%)$ & $1,537(17.4 \%)$ & & $890(30.0 \%)$ & \\
\hline $85+$ & $4,305(8.8 \%)$ & $621(7.0 \%)$ & & $491(16.6 \%)$ & \\
\hline \multicolumn{6}{|l|}{ Albumin, $g / l$} \\
\hline$<30$ & $2,945(6.8 \%)$ & $1,132(13.6 \%)$ & \multirow[t]{6}{*}{$<0.001$} & $382(13.4 \%)$ & \multirow[t]{6}{*}{$<0.001$} \\
\hline $30-35$ & $6,857(15.8 \%)$ & $1,598(19.2 \%)$ & & $584(20.5 \%)$ & \\
\hline $36-38$ & $8,039(18.6 \%)$ & $1,478(17.8 \%)$ & & $591(20.7 \%)$ & \\
\hline $39-42$ & $13,654(31.6 \%)$ & $2,125(25.5 \%)$ & & $767(26.9 \%)$ & \\
\hline $43-45$ & $7,275(16.8 \%)$ & $1,126(13.5 \%)$ & & $335(11.8 \%)$ & \\
\hline $46+$ & $4,499(10.4 \%)$ & $867(10.4 \%)$ & & $191(6.7 \%)$ & \\
\hline \multicolumn{6}{|l|}{ Disabling score } \\
\hline 0 & $5,903(12.0 \%)$ & $894(10.1 \%)$ & \multirow[t]{5}{*}{$<0.001$} & $114(3.8 \%)$ & \multirow[t]{5}{*}{$<0.001$} \\
\hline 1 & $12,286(25.0 \%)$ & $2,178(24.7 \%)$ & & $414(14.0 \%)$ & \\
\hline 2 & $14,187(28.9 \%)$ & $2,783(31.6 \%)$ & & $730(24.6 \%)$ & \\
\hline 3 & $10,002(20.4 \%)$ & $1,885(21.4 \%)$ & & $864(29.1 \%)$ & \\
\hline 4 & $6,709(13.7 \%)$ & $1,071(12.2 \%)$ & & $844(28.5 \%)$ & \\
\hline \multicolumn{6}{|l|}{ Charlson index } \\
\hline 0 & $22,630(46.1 \%)$ & $4,368(49.6 \%)$ & \multirow[t]{3}{*}{$<0.001$} & $716(24.1 \%)$ & \multirow[t]{3}{*}{$<0.001$} \\
\hline 1 & $13,451(27.4 \%)$ & $2,348(26.6 \%)$ & & $723(24.4 \%)$ & \\
\hline$\geq 2$ & $13,006(26.5 \%)$ & $2,095(23.8 \%)$ & & $1,527(51.5 \%)$ & \\
\hline
\end{tabular}

ahypokalaemia vs normokalaemia; bhyperkalaemia vs normokalaemia. LOS = length of stay; SD = standard deviation.

disabling disease score and sepsis status, the fully adjusted OR for in-hospital mortality was 1.30 (95\% CI 1.16-1.45; $\mathrm{p}<0.001)$ (Table 2). The model had an AUROC of 0.86 to predict 30 -day in-hospital mortality (95\% CI 0.85-0.86). The respective in-hospital mortalities at presentation cut-offs of $<2.5, \geq 2.5$ $<3.0, \geq 3.0<3.5$, and $\geq 3.5 \mathrm{mmol} / \mathrm{l}$, were $12.1,7.7,4.3$ and $4.7 \%$ respectively. The OR for in-hospital mortality in those with serum potassium $<3.0$ and $<2.5 \mathrm{mmol} / 1$ were 1.73 (95\% CI

\section{Table 2. Univariate and multivariate mortality by potassium status.}

\begin{tabular}{|c|c|c|c|c|c|c|}
\hline Factor & $\begin{array}{l}\text { Hypokalaemia vs } \\
\text { normokalaemia }\end{array}$ & $95 \% \mathrm{CI}^{\mathrm{a}}$ & $p$ value $^{a}$ & $\begin{array}{l}\text { Hyperkalaemia vs } \\
\text { normokalaemia }\end{array}$ & $95 \% \mathrm{CI}^{\mathrm{b}}$ & $p$ value ${ }^{b}$ \\
\hline Univariate OR for mortality & 1.29 & $1.16-1.43$ & $<0.001$ & 5.20 & $4.70-5.70$ & $<0.001$ \\
\hline Multivariate OR for mortality & 1.30 & $1.16-1.45$ & $<0.001$ & 2.22 & $2.00-2.48$ & $<0.001$ \\
\hline
\end{tabular}




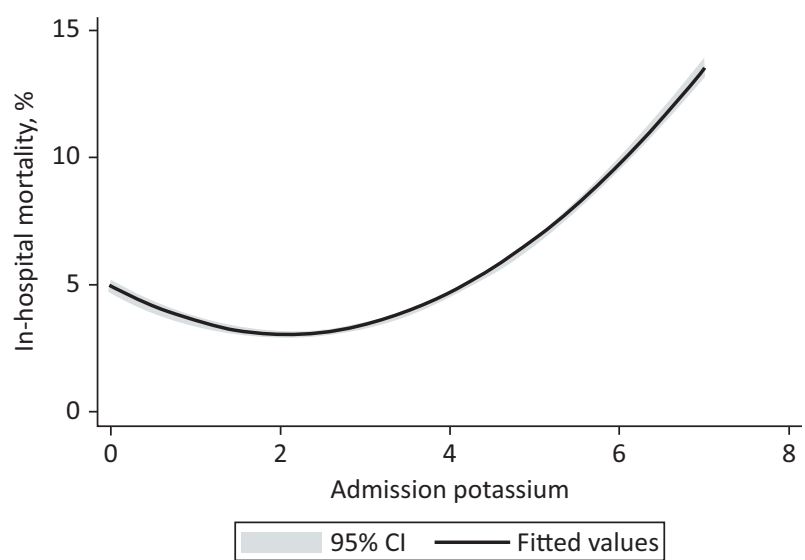

Fig 1. Quadratic plot (with $95 \% \mathrm{CI}$ ) demonstrating the relationship between presenting potassium and 30-day in-hospital mortality. $\mathrm{CI}=$ confidence interval.

$1.41-2.11 ; \mathrm{p}<0.001)$ and $2.34(95 \%$ CI $1.95-2.82 ; \mathrm{p}<0.001)$ respectively.

Hypokalaemic patients had a longer LOS (IQR) of 5.8

(2.7-10.6) days compared with normokalaemia of 4.8 (2.0-9.0) days $(\mathrm{p}<0.001)$.

\section{Association of hyperkalaemia with mortality and hospital length of stay}

Hyperkalaemic patients had an increased in-hospital mortality compared with normokalaemic patients (4.0 vs $18.1 \%$; $\mathrm{p}<0.001$ ) (Table 1). The univariate OR for in-hospital mortality for hyperkalaemia versus normokalaemia was 5.2 (95\% CI 4.7-5.7; $\mathrm{p}<0.001)$. The risk at different levels of potassium is demonstrated in Fig 2. The fully adjusted OR for in-hospital mortality was 2.22 (95\% CI 2.00-2.48; $\mathrm{p}<0.001)$. The model had an AUROC of 0.86 to predict in-hospital mortality (95\% CI $0.85-0.86$ ). The in-hospital mortalities at presentation cut-offs of $\geq 4.3<4.7, \geq 4.7<5.2$, and $\geq 5.2 \mathrm{mmol} / \mathrm{l}$, were $5.5,9.0$, and $18.7 \%$ respectively. The univariate OR for in-hospital mortality in the $\geq 4.3<4.7$ and $\geq 4.7<5.2$ ranges were 1.73 (95\% CI 1.51-1.99) and 2.97 (95\% CI 2.53-3.50). The adjusted ORs for these two groups remained independently predictive for in-hospital mortality at 1.16 (95\% CI 1.00-1.35) and 1.58 (95\% CI 1.33-1.89) ( $\mathrm{p}<0.001)$. Hyperkalaemic patients had a longer LOS (IQR) of 6.6 (3.0-11.9) days compared with normokalaemic patients of 4.8 (2.0-9.0) days $(\mathrm{p}<0.001)$.

\section{Causes of hyperkalaemia}

Analysis of predictors of hyperkalaemia suggested a modest contribution of these factors to the overall effect. Significant ORs were present for chronic kidney disease (OR 1.22; 95\% CI $1.20-1.25$ ), diabetes (OR 1.56; 95\% CI 1.46-1.67), and deep venous thrombosis (OR 1.61; 95\% CI 1.39-1.86).

\section{Discussion}

In our study, admission serum potassium was associated with in-hospital mortality with a J-shaped relationship evident.

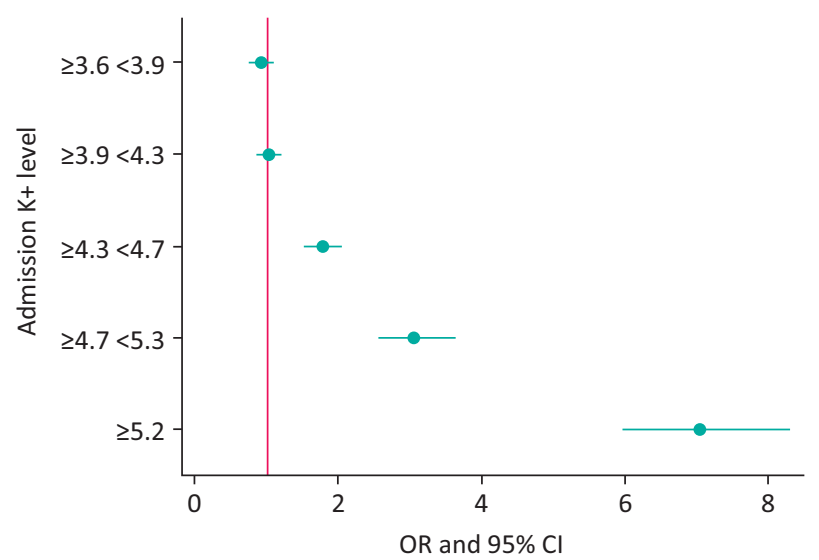

Fig 2. Admission potassium and 30-day in-hospital mortality. $\mathrm{CI}=$ confidence interval.

Hyperkalaemia was a more significant predictor of mortality than hypokalaemia with an OR of 5.2 compared with 1.29 for in-hospital mortality. Strikingly, patients with serum potassium levels in the upper end of the normal range had an increased mortality compared with those in the lower end of the normal range. Patients with serum potassium levels between 4.3 and $4.7 \mathrm{mmol} / \mathrm{l}$ had an OR of 1.73 , and those with serum potassium between 4.7 and $5.2 \mathrm{mmol} / \mathrm{l}$, an OR of 2.97 for death compared with the total population. There was a significant interaction with a number of other variables predictive of outcome, including illness severity and co-morbidity. This attenuated the predictive effect of serum potassium, however it remained significant for all values. In our population, optimum potassium levels appeared to be between 3.0 and $4.3 \mathrm{mmol} / \mathrm{l}$ with values above and below these values associated with an increased mortality.

The results of our study raise a number of clinically important issues. There was no evidence to support the supplementation of serum potassium to high normal levels, and indeed serum potassium at these levels was associated with an increased mortality. In addition there was no increase in mortality for patients with mild decreases in potassium levels. This casts significant doubt on the continued practice of the aggressive supplementation of low-normal serum potassium levels. The management of mildly decreased serum potassium levels must also be reconsidered, and perhaps we should be more cautious in supplementation in these patients in order to avoid potentially fatal hyperkalaemia. By contrast, the extent of the increase in mortality in hyperkalaemic patients is perhaps greater than would have been expected. While the risks of very high potassium values are well known, the majority of our patients with hyperkalaemia had only mild elevations. We have also demonstrated an association of high-normal serum potassium with mortality. Given this data, the approach of being tolerant to mildly elevated potassium values and only treating those with serum potassium $>6.5 \mathrm{mmol} / \mathrm{l}$ or electrocardiographic changes must be called into question. A number of previous studies have assessed the association between serum potassium levels and mortality in acute myocardial infarction. Two recent studies have reported a U-shaped relationship between serum potassium and in- 
hospital and long-term mortality in patients with acute myocardial infarction. ${ }^{6,23}$ Both of these studies showed that serum potassium $<3.5$ or $\geq 4.5 \mathrm{mmol} / \mathrm{l}$ was associated with increased mortality and called into question clinical guidelines recommending serum potassium levels of 4.0-5.0 mmol/1. ${ }^{3,4} \mathrm{~A}$ laboratory-based study found that a lower mortality rate was associated with a serum potassium of $3.4-4.3 \mathrm{mmol} / \mathrm{l}$, however this study was not able to sufficiently control for confounding factors. ${ }^{24}$ In some contrast to the findings of these publications, a recent meta-analysis reported a positive effect of increased potassium intake on blood pressure and the incidence of stroke. ${ }^{25}$ However, this meta-analysis was not able to report on mortality or the effect of intake on serum potassium levels. A recent study evaluating hypokalaemia in acute medical admissions found hazard ratios of 1.56 and 2.17 for 30 -day mortality in patients with serum potassium levels $<3.4$ and $<2.9 \mathrm{mmol} / \mathrm{l}$ respectively, compared with normokalaemia. ${ }^{26}$ Our study adds to these previous studies by demonstrating a J-shaped relationship between serum potassium and mortality in acute general medical admissions.

As with any study, ours has a number of limitations. We have demonstrated an association between serum potassium and in-hospital mortality. This does not imply causation. While we have controlled for multiple other factors affecting mortality, there may be residual unknown confounders affecting our results. We have limited our dataset to those patients discharged or deceased within 30 days of admission in order to avoid an over-representation of long-stay patients admitted for social rather than medical reasons in our results. We believe that this better represents the population on whom decisions will be made in the acute medical setting which was the aim of this paper. The construct of our database enabled us to examine only serum potassium levels at admission, not at the time of death. However, serum potassium levels at admission have been shown to correlate well with ongoing serum potassium levels in the majority of patients. ${ }^{27}$ In addition, the potassium level at the time of death may be unduly influenced by the process of death itself and the modifications and adjustment of medical care often inherent in this.

In conclusion, our study demonstrates a J-shaped association between serum potassium and in-hospital mortality. Highnormal levels of serum potassium were associated with a significant increased mortality.

\section{References}

1 Nyirenda MJ, Tang JI, Padfield PL, Seckl JR. Hyperkalaemia. BMJ 2009;339:b4114.

2 Gennari FJ. Hypokalaemia. N Engl J Med 1998;339:451-8.

3 Antman EM, Anbe DT, Armstrong PW et al. ACC/AHA guidelines for the management of patients with ST-elevation myocardial infarction: a report of the American College of Cardiology/ American Heart Association Task Force on practice guidelines (committee to revise the 1999 guidelines for the management of patients with acute myocardial infarction). Circulation 2004;110:e82-e292.

4 Cohn JN, Kowey PR, Whelton PK, Prisant LM. New guidelines for potassium replacement in clinical practice: a contemporary review by the National Council on Potassium in Clinical Practice. Arch Intern Med 2000;160:2429-36.

5 Macdonald JE, Struthers AD. What is the optimal serum potassium level in cardiovascular patients? J Am Coll Cardiol 2004;43:155-61.
6 Goyal A, Spertus JA, Gosch K et al. Serum potassium levels and mortality in acute myocardial infarction. JAMA 2012;307:157-64.

7 Nordrehaug JE, Johannessen KA, von der Lippe G. Serum potassium concentration as a risk factor of ventricular arrhythmias early in acute myocardial infarction. Circulation 1985;71:645-9.

8 Friedensohn A, Faibel HE, Bairey O, Goldbourt U, Schlesinger Z. Malignant arrhythmias in relation to values of serum potassium in patients with acute myocardial infarction. Int J Cardiol 1991;32:331-8.

9 Kafka H, Langevin L, Armstrong PW. Serum magnesium and potassium in acute myocardial infarction. Influence on ventricular arrhythmias. Arch Intern Med 1987;147:465-9.

10 Conway R, O'Riordan D, Silke B. Long-term outcome of an AMAU - a decade's experience. QJM 2014;107:43-9.

11 O'Loughlin R, Allwright S, Barry J, Kelly A, Teljeur C. Using HIPE data as a research and planning tool: limitations and opportunities. Ir J Med Sci 2005;174:40-5; discussion 52-7.

12 Charlson ME, Pompei P, Ales KL, MacKenzie CR. A new method of classifying prognostic comorbidity in longitudinal studies: development and validation. J Chronic Dis 1987;40:373-83.

13 Conway R, Galvin S, Coveney S, O’Riordan D, Silke B. Deprivation as an outcome determinant in emergency medical admissions. QJM 2013;106:245-51.

14 Manchester Triage Group. Emergency triage, 2nd edn. London: Blackwell Publishing Ltd, 2006.

15 Chotirmall SH, Picardo S, Lyons J et al. Disabling disease codes predict worse outcomes for acute medical admissions. Intern Med J 2014;44:546-53.

16 Ozminkowski RJ, Smith MW, Coffey RM et al. Private payers serving individuals with disabilities and chronic conditions. Washington DC: US Department of Health and Human Services, 2000. Available online at http://aspe.hhs.gov/daltcp/reports/2000/ privpay.htm [Accessed 4 March 2015].

17 Silke B, Kellett J, Rooney T, Bennett K, O'Riordan D. An improved medical admissions risk system using multivariable fractional polynomial logistic regression modelling. QJM 2010;103:23-32.

18 O’Sullivan E, Callely E, O'Riordan D, Bennett K, Silke B. Predicting outcomes in emergency medical admissions - role of laboratory data and co-morbidity. Acute Med 2012;2:59-65.

19 Zeger SL, Liang KY. Longitudinal data analysis for discrete and continuous outcomes. Biometrics 1986;42:121-30.

20 Liang KY, Zeger SL. Longitudinal data analysis using generalized linear models. Biometrika 1986;73:13-22.

21 Hilbe JM. Negative binomial regression, 2 nd edn. Cambridge: Cambridge University Press, 2011.

22 Cameron AC, Trivedi PK. Microeconometrics using Stata. College Station, TX: Stata Press, 2009.

23 Choi JS, Kim YA, Kim HY et al. Relation of serum potassium level to long-term outcomes in patients with acute myocardial infarction. Am J Cardiol 2014;113:1285-90.

24 Solinger AB, Rothman SI. Risks of mortality associated with common laboratory tests: a novel, simple and meaningful way to set decision limits from data available in the electronic medical record. Clin Chem Lab Med 2013;51:1803-13.

25 Aburto NJ, Hanson S, Gutierrez H et al. Effect of increased potassium intake on cardiovascular risk factors and disease: systematic review and meta-analyses. BMJ 2013;346:f1378.

26 Jensen HK, Brabrand M, Vinholt PJ, Hallas J, Lassen AT. Hypokalemia in acute medical patients: risk factors and prognosis. Am J Med 2015;128:60-7.e1.

27 Madias JE, Shah B, Chintalapally G, Chalavarya G, Madias NE. Admission serum potassium in patients with acute myocardial infarction: its correlates and value as a determinant of in-hospital outcome. Chest 2000;118:904-13.

Address for correspondence: Dr B Silke, Department of Internal Medicine, St James's Hospital, Dublin 8, Ireland. Email: bernardsilke@physicians.ie 